\title{
Erratum to: From inspired modeling to creative modeling
}

\author{
Daniel Cohen-Or ${ }^{1} \cdot$ Hao Zhang ${ }^{2}$
}

Published online: 6 April 2016

(C) Springer-Verlag Berlin Heidelberg 2016

\section{Erratum to: Vis Comput (2016) 32:7-14 DOI 10.1007/s00371-015-1193-9}

Figures 1-3 were mixed-up in the original article by accident. The current Fig. 3 is the correct Fig. 1 referenced at the end of the second paragraph in Section 1. The correct Fig. 1 should actually appear on article first page. The current Fig. 1 is the correct Fig. 2.

The correct Figs. 1, 2, and 3 are given below:
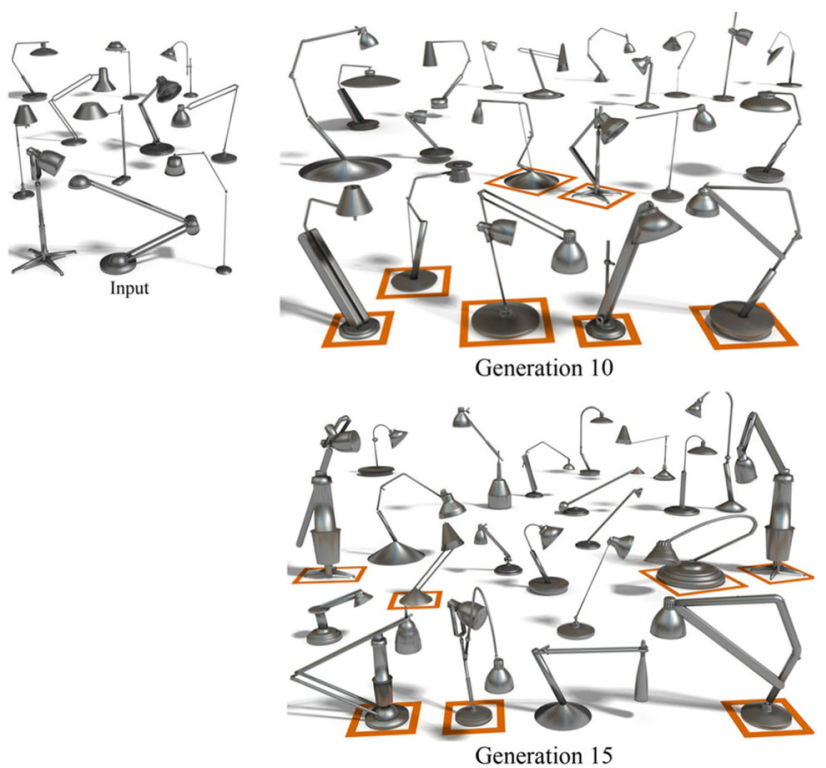

Fig. 1 An evolution-based, example-driven creative modeling tool [45] allows generations of 3D models to be created. The highlighted models exhibit diversity from the input set
The online version of the original article can be found under doi:10.1007/s00371-015-1193-9.

Daniel Cohen-Or

dcor@tau.ac.il

Hao Zhang

haoz@cs.sfu.ca

1 Tel Aviv University, Tel Aviv, Israel

2 Simon Fraser University, Burnaby, Canada 


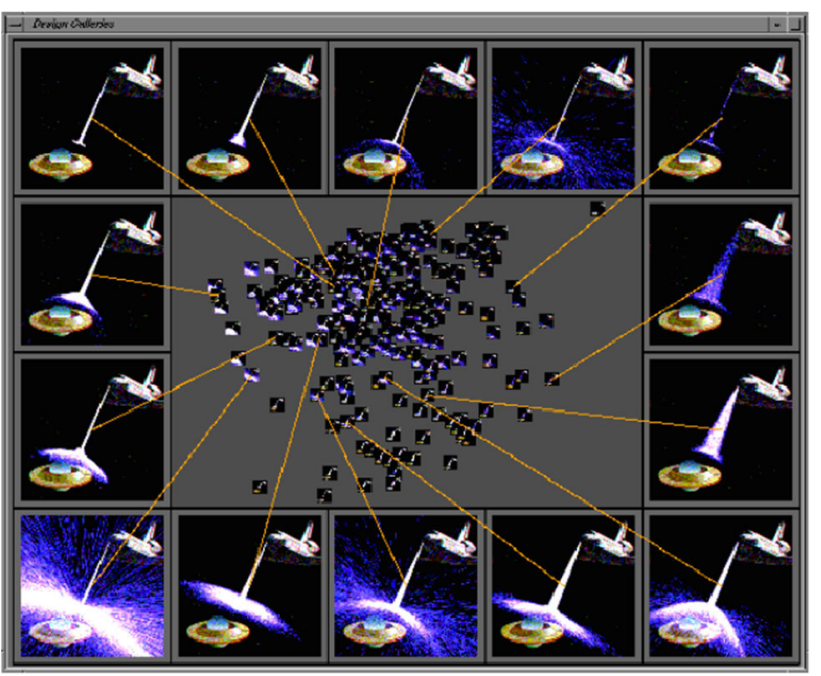

Fig. 2 A design gallery for particle systems (figure taken from [25] with permission). Design alternatives surround a depiction of the design space being explored

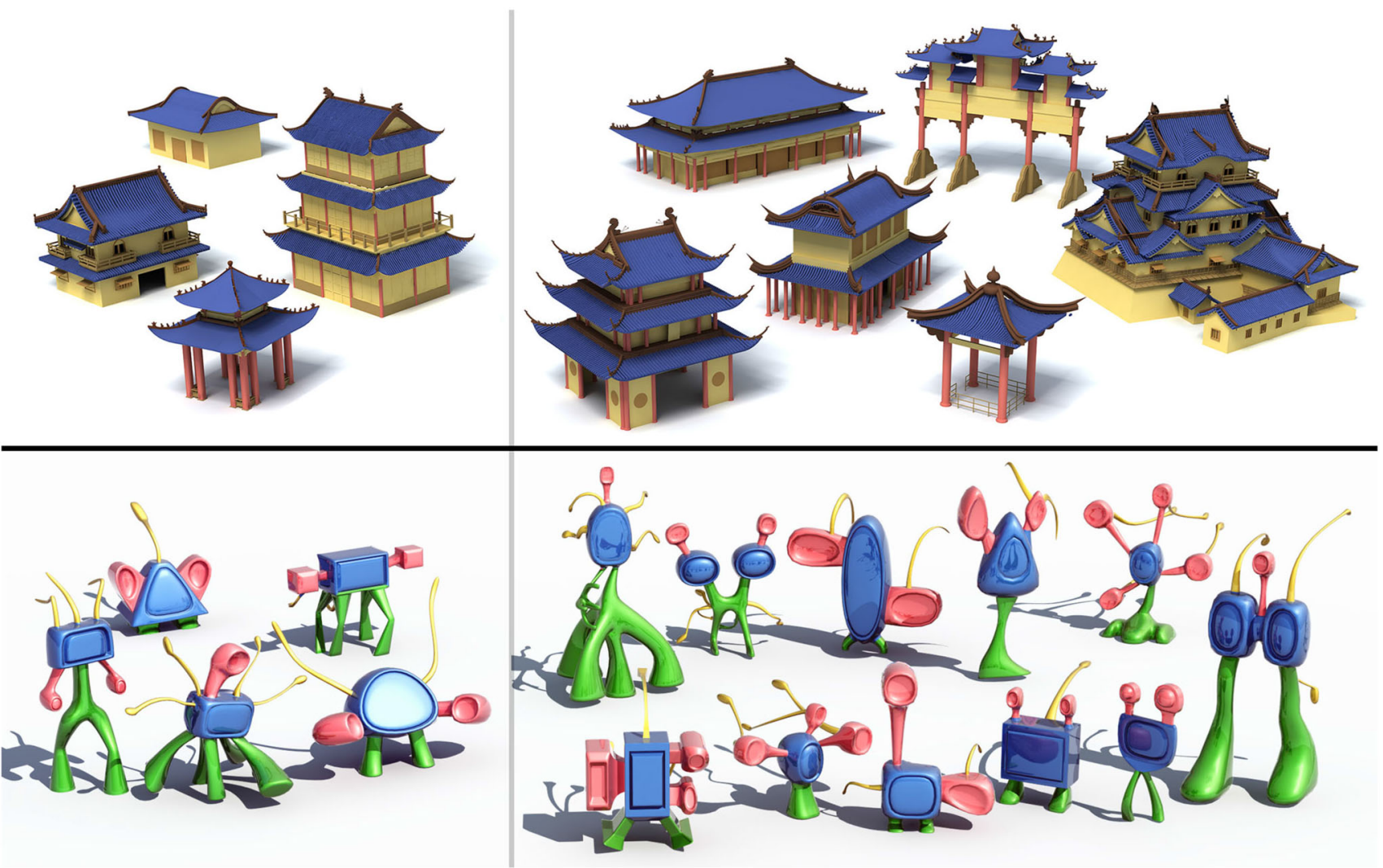

Fig. 3 "More of the same": two sets of example-driven modeling. The examples are shown on the left, and the generated instances are on the right. Courtesy of Jiacheng Ren 